\title{
Chemical Nature of Remineralized Flattened Enamel Surfaces
}

\author{
DAVID J. KRUTCHKOFF* and NATHANIEL H. ROWE
}

Department of Oral Pathology, School of Dentistry, University of Michigan, Ann Arbor, Michigan 48104, USA

Infrared internal reflection spectroscopy (IRS) was used to investigate chemical changes of enamel surface during in vitro remineralization. Specimens were demineralized and exposed either to calcium phosphate-fluoride solutions or to acidulated fuorophosphate. The former showed amorphous phosphate remineralization, and the latter showed surface precipitate interpreted to be calcium fluoride. The significance of previous demineralization time to both forms of remineralization is discussed.

In vitro enamel remineralization has been demonstrated previously by the determination of changes in experimental parameters. Increase in surface hardness after immersion in remineralizing solutions has been proved. $^{1-4}$ Increased radiodensity 5 and negative birefringence ${ }^{6}$ of ground sections of etched enamel have been reported after in vitro remineralization. Recently, the electron probe has been used to demonstrate changes in calcium-phosphorus $(\mathrm{Ca} / \mathrm{P})$ ratios with acid etching and in vitro remineralization. ${ }^{7}$ Experimental evidence indicates that remineralization of enamel may occur in different ways and result in a variety of reaction products. ${ }^{8,9}$

Although there is little doubt that remineralization occurs, 7,10 there is much disagreement about the chemical nature of the resultant enamel surface. In general, analytic methods now used in studies of in vitro remineralization are incapable of molecular characterization. Thus, definitive chemical mapping of remineralization interactions has

This investigation was supported in part by USPHS Research Grant 5 FO3 DE $39678-03$ from the National Institute of Dental Research, National Institutes of Health, Bethesda, Md.

Received for publication August 12, 1970.

- Present address: Health Science Center, University of Louisville, Louisville, Kentucky 40202. not been accomplished. Infrared internal reflection spectroscopy (IRS) recently has been demonstrated to be sensitive and reproducible for analysis of enamel surface in vitro. ${ }^{11}$ This study was an initial attempt to characterize the chemical changes that occur in partially demineralized enamel surfaces subjected to in vitro remineralization.

\section{Materials and Methods}

Sixteen flattened human enamel specimens previously subjected to weak acid demineralization were used. Specimens were enamel slabs prepared from the labial surface of freshly extracted teeth. Each was disked and flattened with garnet strips until a polished facet (approximately 20 to 40 $\mathrm{mm}^{2}$ ) was obtained. Slabs were washed in water and dried in air before the initial pretreatment IRS analysis. Specimens were demineralized in lactic acid-gelatin mixtures ${ }^{12}$ (pH 3.0) for either 5 minutes (samples 1 to 5 ) or 20 minutes (samples 6 to 16). IRS analysis of each specimen was made before and after demineralization, and after remineralization. Base-line (control) spectra were run periodically to test for prism contaminants. All multiple reflection analyses were done with $2 \mathrm{~mm}$ KRS-5 prisms in an IRS accessory.* Samples were scanned from 7 to 25 micrometers $(\mu \mathrm{m})$ in an IR spectrophotometer $\dot{\dagger}$

Effects of two different remineralizing procedures were studied. Samples were separated into two groups; odd numbered specimens were assigned to group 1 , and even numbered specimens to group 2. Group 1 specimens were remineralized by the method

* Wilks Model 45 Micro-ATR, Wilks Scientific Corp., So. Norwalk, Conn.

$\dagger$ Perkin-Elmer 337 Spectrophotometer, Downers Grove, 111. 
of Pigman, Cueto, and Baugh. ${ }^{2}$ Calcium phosphate was dissolved in $0.1 \mathrm{M} \mathrm{HCl}$. Sodium fluoride was added $(1.89 \mathrm{gm} / \mathrm{liter})$, dissolved, and the solution brought to $\mathrm{pH}$ 7.3 by titration with $0.45 N \mathrm{KOH}$. In this study, sodium fluoride was substituted for potassium fluoride, which was used by Pigman, Cueto, and Baugh. ${ }^{2}$ Group 1 specimens were placed in remineralizing solution, heated to $37 \mathrm{C}$, and stirred magnetically with a Teflon-coated rod for six hours. Specimens were rinsed for 48 hours in fresh distilled water, dried in air, and analyzed by IRS. Group 2 specimens were remineralized in acidulated fluorophosphate (AFP) solutions by the method of Aasenden, Brudevold, and McCann. ${ }^{13}$ Specimens were immersed in a solution of $0.1 M$ phosphoric acid and $1.2 \%$ fluoride, and stirred for three minutes. Specimens were rinsed in magnetically stirred, deionized water for 24 hours, dried in air, and analyzed by IRS.

\section{Results}

IRS spectra of remineralized group 1 specimens that had been demineralized for five minutes showed partial reversal of peak resolution produced during demineralization (Fig 1). The $v_{3}$ phosphorous-oxygen absorption maximum (asymmetric stretch) ${ }^{14}$ shifted from approximately $15 \mathrm{~cm}^{-1}$ to 1,015 $\mathrm{cm}^{-1}$ (low energy frequency). A corresponding increase in absorption at about 930 to $970 \mathrm{~cm}^{-1}$ also was noted. Spectra of remineralized specimens showed a gradual diminishing of absorption from the maximum of $1,015 \mathrm{~cm}^{-1}$ to a plateau at $700 \mathrm{~cm}^{-1}$, contrasted by the abrupt change observed in spectra of specimens before remineralization. The lower energy phosphorous-oxygen $v_{4}$ segment (asymmetric bend) became broader, similar to its appearance before demineralization. Overall spectral changes were increased blunting of low energy $v_{3}$ and $v_{4}$ phosphorous-oxygen absorption bands that resulted in loss of peak definition and spectral resolution. IRS analysis of group 1 specimens that had been demineralized for 20 minutes and remineralized, showed little or no surface change from the demineralized state (Fig 2). Absorption peaks remained sharp and did not shift in frequency.

Group 2 specimens showed consistent spectral changes, regardless of previous demineralization time. IRS spectra of the speci-

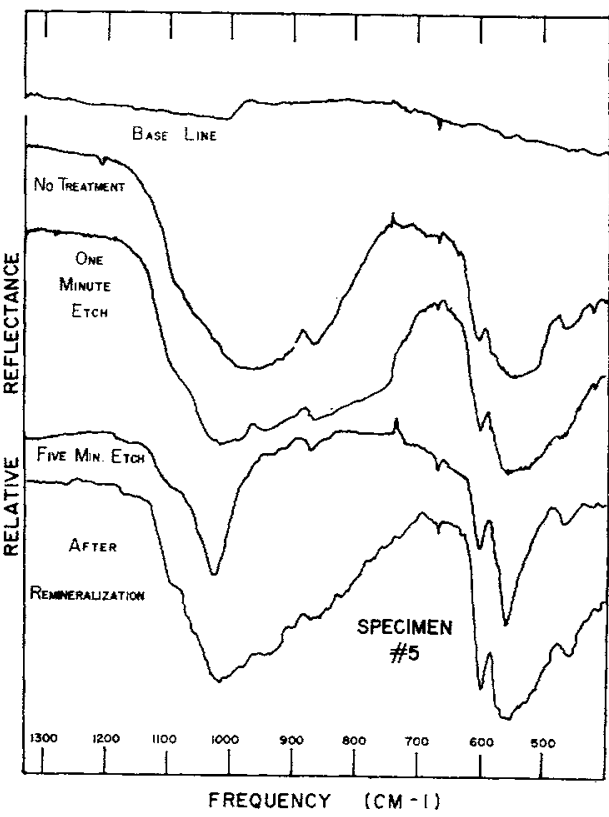

FIG 1.-Composite IRS spectra of representative enamel specimens remineralized in neutral ( $\mathrm{pH}$ 7.3) solution after five minute demineralization. Note partial loss of peak resolution and broad, ill-defined absorption after remineralization. This was interpreted as evidence for amorphous phosphate remineralization. Spectra of additional specimens in this subgroup exhibited similar features.

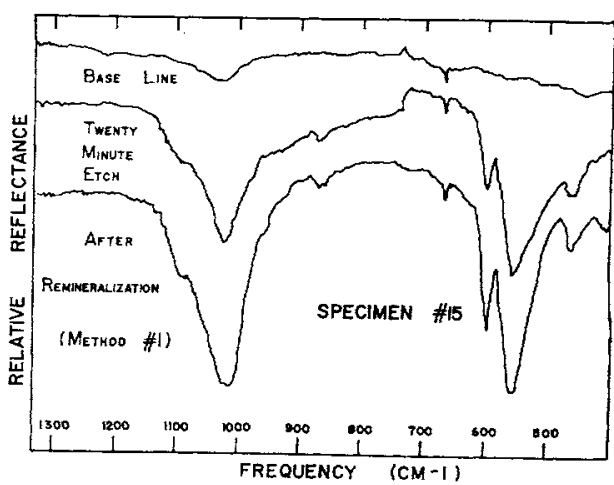

FIG 2.-IRS spectra of 20 minute demineralized specimen, before and after neutral $(\mathrm{pH}$ 7.3) remineralization. Similarity of the two spectra indicates lack of surface remineralization when group 1 specimens were demineralized previously for longer periods. Spectra of other specimens in this subgroup showed similar features. 
mens before remineralization showed increased resolution with improved definition of absorption peaks. After the AFP treatment that followed, absorption diminished almost to the level of absorption by baseline spectra (Fig 3). Some indications of absorption were present, but resolution was inadequate, and absorption peaks could not be identified with certainty.

\section{Discussion}

IRS spectra of group 1 specimens that had been demineralized for five minutes demonstrated evidence of noncrystalline remineralization. Loss of peak definition and frequency shifts to lower energy levels suggest surface acquisition of amorphous phosphate material (Fig 1). In contrast, specimens that had been demineralized for 20 minutes did not show surface evidence of phosphate remineralization (Fig 2). The

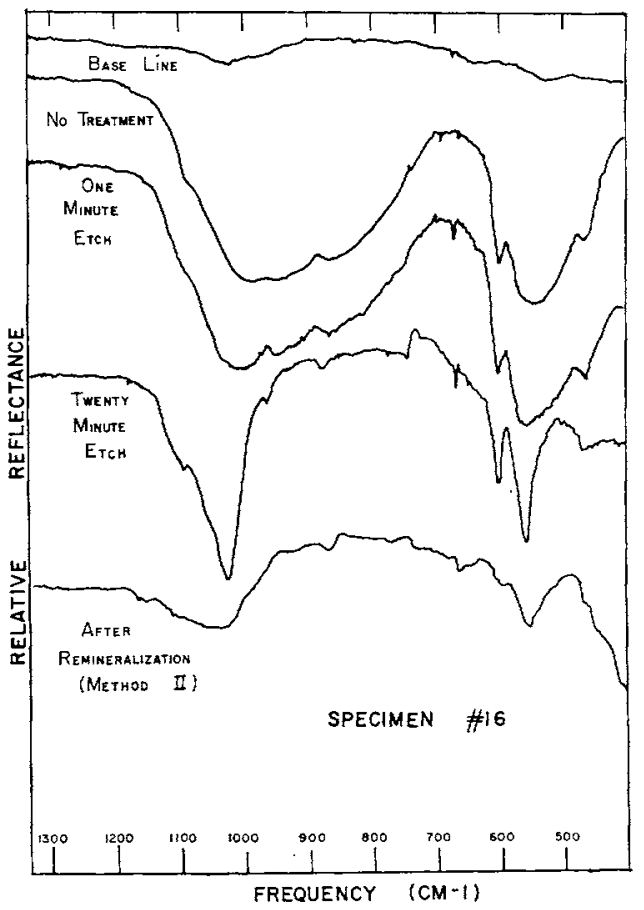

FIG 3.-Composite IRS spectra of enamel specimen subjected to AFP remineralizing solutions after 20 minutes of demineralization. Note strong diminution of absorption and precipitous absorption from 500 to $400 \mathrm{~cm}^{-1}$ after remineralization. Spectra of other specimens in this group, both 5 and 20 minute demineralizations, exhibited similar features. most likely explanation is that 20 minute demineralization resulted in morphologic degradation of enamel to deeper levels of the specimen surface. From examination by scanning electron microscopy (SEM) of fractured carious enamel surface, Poole and Silverstone ${ }^{15}$ have estimated depth of honeycomb craters to be as great as $20 \mu \mathrm{m}$. Remineralization changes at $20 \mu \mathrm{m}$ or deeper would be beyond the deepest penetration of the evanescent wave, and beyond the range of IRS surface analysis. ${ }^{16}$ In contrast, specimens demineralized for five minutes would be altered to relatively shallow depths. Precipitation of amorphous phosphate in superficial enamel microstructure would be detectable by IRS methods (ie, close proximity of prism surface to superficially deposited precipitate). Thus, freshly precipitated amorphous phosphate was detected in specimens that had been demineralized for short periods of time.

Enamel specimens remineralized in AFP solutions showed surface changes that appeared to be independent of previous demineralization time (Fig 3 ). IRS spectra showed a decided decrease in spectral resolution at absorbing frequencies. There was no change in frequency of absorption peaks, but they were considerably diminished in amplitude. Strong absorption was noted from 500 to $400 \mathrm{~cm}^{-1}$ after remineralization. Comparison of IRS spectra of AFP-treated enamel and optical grade calcium fluoride $\left(\mathrm{CaF}_{2}\right)^{*}$ showed strong similarity (Fig 4). Resemblance of $\mathrm{CaF}_{2}$ spectra to that of the base line (no sample) demonstrated the expected infrared transparency of calcium fluoride from $4,000 \mathrm{~cm}^{-1}$ to $500 \mathrm{~cm}^{-1}$. At 500 $\mathrm{cm}^{-1}$, calcium fluoride suddenly began absorption that continued throughout the spectral scan. Similar absorption was observed in IRS spectra of specimens treated with AFP (Fig 4). The observed decrease of apatitic absorption after AFP treatment suggests that underlying mineral was masked by a surface precipitate that was transparent in the absorbing infrared range. Spectral evidence suggests the presence of a calcium fluoride precipitate on the surface of demineralized enamel specimens that were treated with AFP solutions.

IRS data reported here suggest that prod-

* Peter Stern and William Higuchi, Department of Pharmaceutical Chemistry, University of Michigan, Ann Arbor, Mich. 


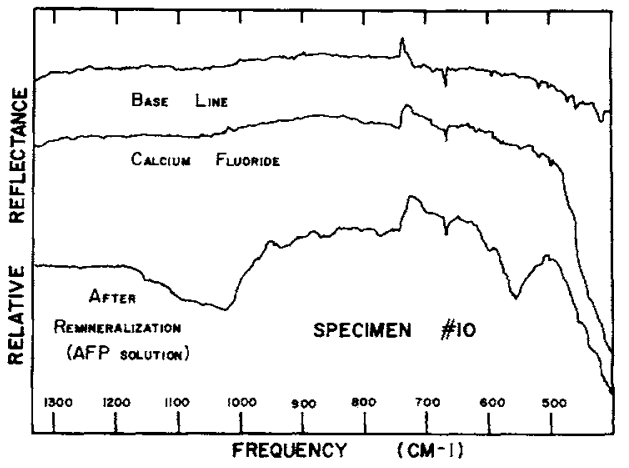

FIG 4.-IRS spectrum of pure calcium fluoride shows transparency (ie, lack of absorption) to infrared energy, up to approximately $500 \mathrm{~cm}^{-1}$. Enamel specimens remineralized in AFP solution exhibit diminution of absorption up to approximately $500 \mathrm{~cm}^{-1}$, followed by precipitous absorption similar to that seen with $\mathrm{CaF}_{2}$. These observations were interpreted to indicate calcium fluoride precipitation after AFP remineralization.

ucts of remineralization differed and depended on the composition and the $\mathrm{pH}$ of the remineralizing solution. Remineralization at neutral or slightly basic $\mathrm{pH}$ (saturated calcium phosphate solutions that contained fluoride) resulted in noncrystalline phosphate precipitates. This reaction previously has been shown to increase surface hardness in vitro, ${ }^{3,4}$ and probably resembles in vivo remineralization when calcium and phosphate are in neutral excess in the immediate environment of the enamel surface. In contrast, treatment of demineralized enamel with AFP solution yielded spectral evidence interpreted as surface precipitation of calcium fluoride. This precipitation apparently was not related to previous demineralization time. Studies that used X-ray diffraction, ${ }^{17-20}$ electron microscopy, ${ }^{20,21}$ and chemical analysis ${ }^{22-26}$ have shown that calcium fluoride is a product of the enamel-AFP interaction. IRS evidence supports a previous proposal ${ }^{8,18}$ that phosphates of AFP solutions do not suppress significantly the formation of calcium fluoride, as proposed by other investigators. ${ }^{27}$ Since calcium ions were not present in experimental AFP solutions used in this study, calcium was dissolved from underlying enamel and precipitated rapidly as relatively acid-stable calcium fiuoride. ${ }^{28}$ The precipitate was relatively tenacious, because it was detected by IRS after 24 hours of agitated water rinse.

\section{Conclusions}

The surfaces of most demineralized enamel specimens analyzed in this study underwent chemical changes after in vitro treatment with remineralizing solutions. Chemical changes were uniform within the various groups, differing between groups on the basis of experimental conditions. With neutral saturated calcium phosphate solutions that contained fluoride, remineralization occurred in enamel as relatively noncrystalline calcium phosphates. The extent of previous weak acid demineralization was a significant variable in determining the nature of enamel surface after this method of remineralization. In contrast, AFP remineralization resulted in a surface precipitate thought to be calcium fluoride. This surface precipitate occurred independently of previous demineralization time. IRS was useful in the determination of in vitro remineralization of flattened enamel surfaces.

\section{References}

1. Koulourides, T.: Remineralization of Enamel and Dentin, Dent Clin $N$ Amer, 485-497, 1962.

2. Pigman, W.; Cueto, H.; and Baugh, D.: Conditions Affecting the Rehardening of Softened Enamel, J Dent Res 43:1187-1195, 1964.

3. Koulourides, T.; Feagin, F.; and Pigman, W.: Remineralization of Dental Enamel by Saliva In Vitro, Ann NY Acad Sci 131: 685-693, 1965.

4. Koulourides, T.; Cueto, H.; and Pigman, W.: Rehardening of the Softened Enamel Surfaces of Teeth by Solutions of Calcium Phosphates, Nature 189:226-227, 1961.

5. Amprino, R., and Camanni, F.: Historadiographic and Autoradiographic $\mathrm{Re}-$ searches of Hard Dental Tissues, Acta Anat 28:217-258, 1956.

6. JohansSon, B.: Remineralization of Slightly Etched Enamel, J Dent Res 44:64-70, 1965.

7. WEr, S.H.Y.: Electron Microprobe Analyses of the Remineralization of Enamel, $J$ Dent Res 49:621-625, 1970.

8. WEI, S.H.Y., and Forbes, W.C.: X-Ray Diffraction Analyses of the Reactions Between Intact and Powdered Enamel and Several Fluoride Solutions, $J$ Dent Res 47: 471-477, 1968.

9. WEI, S.H.Y.; KAQUELLER, J.C.; and MASSLER, M.: Remineralization of Carious Dentin, J Dent Res 47:381-391, 1968. 
10. WEI, S.H.Y.: Remineralization of Enamel and Dentin-A Review, $J$ Dent Child 34: 444-451, 1967.

11. KRUTChKoFF, D.J.; Rowe, N.H.; and MARK, H.B., Jr.: Infrared Internal Reflection Spectroscopy of Human Enamel Surface, Arch Oral Biol 16:161-175, 1971.

12. Silverstone, L.M.: The Surface Zone in Caries and in Caries-Like Lesions Produced In Vitro, Brit Dent J 125:145-157, 1968.

13. Aasenden, R.; Brudevold, F.; and McCANN, H.G.: The Response of Intact and Experimentally Altered Enamel to Topical Fluoride, Arch Oral Biol 13:543-552, 1968.

14. Stutman, J.M.; TERMINE, J.D.; and PosNer, A.S.: Vibrational Spectra of the Phosphate Ion in Some Calcium Phosphates, Trans NY Acad Sci 27:669-675, 1965.

15. Poole, D.F.G., and Silverstone, L.M.: Observations with the Scanning Electron Microscope on Trauma-Induced Microcavities in Human Enamel, Arch Oral Biol 14:1323-1329, 1969.

16. HARRICK, N.J.: Internal Reflection Spectroscopy, New York: Wiley \& Sons, 1965, pp $1-62$.

17. Frazier, P.D.: Electron Probe Analysis of Human Teeth Ca-P Ratios in Incipient Carious Lesions, Arch Oral Biol 12:25-34, 1967.

18. Frazier, P.D., and Engen, D.W.: X-Ray Diffraction Study of the Reaction of Acidulated Fluoride with Powdered Enamel, J Dent Res 45:1114-1148, 1948.

19. Gerould, C.H.: Electron Microscopic Study of the Mechanisms of Fluoride Deposition in Teeth, $J$ Dent Res 24:223-233, 1954.
20. Scott, D.B.; PICARD, R.G.; and Wyckoff, R.W.G.: Studies of the Action of Sodium Fluoride on Human Enamel by Electron Microscopy and Electron Diffraction, $P u b$ lic Health Rep 65:43-56, 1950.

21. Fosdick, L.S., and Hutchinson, A.C.: The Mechanism of Caries of Dental Enamel, Ann NY Acad Sci 131:758-770, 1965.

22. DeShazer, D.O., and Schwartz, C.J.: The Formations of Calcium Fluoride on the Surface of Hydroxyapatite After Treatment with Acidic Fluoride-Phosphate Solutions, Arch Oral Biol 12:1071-1075, 1967.

23. LEACH, S.A.: Reactions of Fluoride with Powdered Enamel and Dentin, $J$ Dent Res 34:59, 1959.

24. MCCANN, H.G.: Reaction of Fluoride Ion with Hydroxyapatite, $J$ Biol Chem 201: 247-259, 1953.

25. McCanN, H.G.: The Solubility of Fluorapatite and its Relationship to that of Calcium Fluoride, Arch Oral Biol 13:9871001, 1968.

26. McCanN, H.G., and Bullock, F.A.: Reactions of Fluoride Ions with Powdered Enamel and Dentin, $J$ Dent Res 34:59-67, 1955.

27. Brudevold, F.; Savory, A.; Gardner, D.E.; SPINElil, M.; and SpIERS, R.: A Study of Acidulated Fluoride Solutions: I. In Vitro Effects on Enamel, Arch Oral Biol 8:167-177, 1963.

28. Francis, M.D.; Gray, J.A.; and GriebSTEIN, W.J.: The Formation and Influence of Surface Phases on Calcium Phosphate Solids, in STAPLE, P.H. (ed): Advances in Oral Biology, New York: Academic Press, vol 3, 1968, pp 83-120. 